University of Nebraska - Lincoln

DigitalCommons@University of Nebraska - Lincoln

Faculty Publications - Department of

Philosophy

Philosophy, Department of

2005

Are Dispositions Causally Relevant?

Jennifer McKitrick

University of Nebraska-Lincoln, jmckitrick2@unl.edu

Follow this and additional works at: https://digitalcommons.unl.edu/philosfacpub

McKitrick, Jennifer, "Are Dispositions Causally Relevant?" (2005). Faculty Publications - Department of Philosophy. 7.

https://digitalcommons.unl.edu/philosfacpub/7

This Article is brought to you for free and open access by the Philosophy, Department of at DigitalCommons@University of Nebraska - Lincoln. It has been accepted for inclusion in Faculty Publications Department of Philosophy by an authorized administrator of DigitalCommons@University of Nebraska - Lincoln. 


\title{
Are Dispositions Causally Relevant?
}

\author{
Jennifer McKitrick \\ University of Nebraska-Lincoln
}

\begin{abstract}
To determine whether dispositions are causally relevant, we have to get clear about what causal relevance is. Several characteristics of causal relevance have been suggested, including Explanatory Power, Counterfactual Dependence, Lawfulness, Exclusion, Independence, and Minimal Sufficiency. Different accounts will yield different answers about the causal relevance of dispositions. However, accounts of causal relevance that are the most plausible, for independent reasons, render the verdict that dispositions are causally relevant.
\end{abstract}

Are dispositions causally relevant to their manifestations? According to some philosophers, dispositions are inert, impotent, causally irrelevant, and inefficacious (Block 1990; Kim 1988; Prior et al. 1982). Presumably, other properties do not suffer these inadequacies, but have some relation to events that dispositions lack. Why would anyone think such a thing? The following arguments are typical:

The Analyticity Argument (Block 1990; Dardis 1993; Jackson 1995):

1. "Being fragile" means being prone to break.

2. Therefore, fragility is non-contingently connected to breaking.

3 A cause is only contingently connected to its effect.

4. Therefore, fragility is not causally relevant to breaking.

The Exclusion Argument (Kim 1990; Prior, Pargetter, and Jackson 1982):

1. The crystalline structure of the glass and the striking (plus background circumstances) constitute a complete causal explanation of the breaking.

2. Therefore, there is no causal/explanatory work for the glass's fragility to do.

3. Therefore, fragility is not causally relevant to breaking.

One may find fault with these arguments on a number of grounds. However, in this paper I want to explore the implicit assumptions these arguments make about the nature of causal relevance. What conditions or constraints should we use in deciding whether a property is causally relevant? I will consider several candidate conditions on causal relevance and consider 
what they mean for dispositions. The upshot is that the most plausible accounts of causal relevance will count dispositions as causally relevant.

Causal relevance is one thread in a web of interrelated concepts, which includes causation, events, properties, laws of nature, necessity, and possibility. I am not going to tease all of these concepts apart, or justify all of my assumptions. I am assuming that causation is a relation between events, which I think of as space-time regions and their contents. Beyond that, I do not take myself to be assuming or offering any particular account of causation. Many conditions of causal relevance appeal to laws of nature, and again, no particular account of laws is assumed.

What do I mean by "causal relevance"? Examples convey the intuitive idea. Johnny throws a baseball and it shatters a windowpane. Even if we assume that the throwing event caused the breaking event, there is a further question we may consider. Which properties of the cause are relevant to the effect? The mass and the velocity of the ball seem like prime candidates for being relevant to the window breaking. However, the color of the ball is unlikely to be relevant.

In the first instance causal relevance is a relation between a property and an effect. So, we want an account of statements of the form: "Property F is causally relevant to event e." $\mathrm{F}$ is a property of the cause of e or of some part of the cause. I move freely between talk of properties of the event, and properties of part of the event as candidate causally relevant properties. If you prefer to restrict causal relevance to properties of events, you can think of an object's property $\mathrm{F}$ in terms of an event's property of involving an $\mathrm{F}$ object (Braun 1995).

"Causal relevance" is something of a philosophical term of art with no standard usage. Some philosophers take it to relate different relata-a property and a property, an event and an event, various combinations of properties and events. Philosophers also employ different terminology: "causally potent," "causally responsible," "explanatorily relevant," "causally efficacious," etc. Some philosophers make subtle distinctions between these terms (Block 1995; Fodor 1989; Hitchcock 1993; Jackson 1995; Jackson and Pettit 1990; Lepore and Loewer 1987; MacDonald and MacDonald 1995; Segal and Sober 1991). Perhaps we need to distinguish different relations that properties of causes can have to effects. Nevertheless, people have intuitions about what is and is not causally relevant, and they talk as if they disagree about whether dispositions are causally relevant (Jackson and Pettit 1990; Segal and Sober 1991). It seems as though there is some root notion people are concerned about, and this is more than a mere verbal dispute. One way to break through the jargon and get to the crux of the issue is to ask: What relation is supposed to hold between the mass of the ball and the 
window breaking, but not the color of the ball and the window breaking? Does this same relation hold between the fragility of the glass, and the window breaking?

I assume the following basic minimal necessary condition on causal relevance: for a property to be causally relevant to an event, it must be a property of the cause of that event.

If $\mathrm{F}$ is causally relevant to $\mathrm{e}$, then there is some event $\mathrm{c}$ such that $\mathrm{c}$ causes $\mathrm{e}$, and $\mathrm{c}$ has $\mathrm{F}$.

Of course, this condition is not sufficient. It does not distinguish between mass of ball and color of ball. Further conditions need to be added to get a complete account. We want to screen off the causally irrelevant properties. That is what these further conditions purport to do. I suppose all of them are intended to be necessary conditions, and some set of them, plus the minimal necessary condition above, would ideally make for necessary and sufficient conditions. Failures of sufficiency do not show that the condition should be rejected, but perhaps augmented with further conditions. If a condition is unnecessary, that is prima facie reason to reject it. I argue that the conditions that screen off dispositions are unnecessary.

\section{Explanatory Conditions (Dretske 1988; Kim 1988)}

A causally relevant property has some explanatory power. If $\mathrm{F}$ is causally relevant to e, then F explains e. Well, what does that mean? Perhaps F is apt for being mentioned in a causal explanation of why e occurred. In other words, F is part of a satisfying answer to the question "why did e occur?." Call this the Weak Explanatory Condition.

Notice that dispositions are apt for being mentioned in explanations of their manifestations. Why did the glass break? It was fragile. Moliere not withstanding, that would be a satisfying explanation in many contexts (Moliere 1935). Why did I get a shock? Because I was touching something that was electrically conductive. Why is she so quiet? She's shy.

But we can concoct stories in which apparently irrelevant properties are mentioned in explanations which satisfy their audiences. Suppose Johnny has a white baseball and a yellow foam-rubber ball. Jane asks "why did the window break?" and she receives the answer "because Johnny threw the white ball." Jane has the relevant background information, and so this explanation satisfies her. But we were supposing that the color of the ball was not relevant to the window breaking. ${ }^{1}$ 
We can concoct stories like this, because various pragmatic considerations determine whether an answer to a why question is satisfactory. What background knowledge does the audience have? What are their interests? Proponents of explanatory conditions want to by-pass these epistemic, pragmatic considerations. Perhaps we could say that if $\mathrm{F}$ is causally relevant to e, then $\mathrm{F}$ would be mentioned in an ideal explanation of why e occurred. Alternatively, there is no complete, true explanation of e that fails to mention F. Call this the Strong Explanatory Condition. If complete, true, ideal explanations of events do not mention dispositions, this view would have the consequence that dispositions are not causally relevant.

However, I am skeptical of the idea of an audience-independent "ideal explanation." Different explanations have different degrees of complexity and detail, and operate at different "levels." There can be a true, complete explanation of an event that does not mention a causally relevant property, because the explanation operates at a level of description at which mentioning the property is not appropriate or salient.

Some people think that explanatory relations are out there in the world. A property can explain some event even if no one knows about it. But even the realist about explanation does not hold that explanatory facts are brute facts. If $\mathrm{F}$ explains e, that must be in virtue of some other relation that $\mathrm{F}$ and e have. Maybe F explains e because F is causally relevant to e. So, this condition on causal relevance seems to put the cart before the horse.

\section{The Counterfactual Dependence Condition (Horgan 1989; Lepore and Loewer 1987, 1989; Ruben 1994)}

One might say that the important difference between the velocity of the ball and its color, vis-a-vis the breaking, is the following: If the throw had not been so fast, then the window would not have broken; however, even if the ball had not been white, the window would have broken just the same. The effect depends on the instantiation of the causally relevant property. That is to say:

If $\mathrm{F}$ is causally relevant to e,

then if $\mathrm{c}$ did not have $\mathrm{F}$, then e would not have occurred.

Notice dispositions pass this test. If the glass were not fragile, it would not have broken.

Admittedly, this account has some difficulties. One oft noted problem is over-determination or pre-emption. Maybe the glass would have broken due to some other cause. Suppose there was another baseball heading for the win- 
dow. Then, even if the throw were not fast enough, the window still would have broken. As we shall see, over-determination will cause problems for other accounts of causal relevance. I think the Counterfactual Dependence Condition can get around over-determination by other events by making the following alteration.

If $\mathrm{F}$ is causally relevant to e,

then if $\mathrm{c}$ did not have $\mathrm{F}$, then $\mathrm{c}$ would not have caused e.

If the throw had not been so fast, then it would not have broken the glass. This would be true even if something else would have broken the glass instead. Again, dispositions pass this test. If the wire had not been conductive, then touching the wire would not have caused a shock. ${ }^{2}$

The Counterfactual Dependence Condition still has a problem with overdetermining properties. Suppose I notice a bright flashing light. My noticing it is over-determined by the brightness and the flashing. It seems that both the brightness and the flashing are causally relevant to my noticing the light. However, the associated counterfactuals are false. The account tells us that, since the brightness is causally relevant, if the light were not bright, the bright flashing light would not have gotten my attention, but that is false.

Granted, there are unsolved problems for this account. As others have discussed, the Counterfactual Dependence Condition has trouble with closely related properties (traveling $60 \mathrm{mph}$ and traveling $59 \mathrm{mph}$ ) and co-extensive properties (electrical conductivity and thermal conductivity) (Block 1990; Braun 1995). Nevertheless, there is something to the idea that effects are counterfactually dependent on the causally relevant properties of their causes. And while philosophers tend to turn to counterfactual dependence as a test of causal relevance, they less frequently observe that dispositions pass this test (Allen 1995; Heil and Mele 1991).

\section{The Increased Probability Condition}

Drawing on probabilistic accounts of causation (Hitchcock 1993), it occurred to me that we could analyze causal relevance in the following way. If a property is causally relevant it increases the probability of the effect.

If $\mathrm{F}$ is causally relevant to $\mathrm{e}$, then $\mathscr{P}(\mathrm{e} / \mathrm{cF})>\mathscr{P}(\mathrm{e} / \mathrm{c} \sim \mathrm{F})$.

The probability of the window breaking when the trajectory of the throw is towards the window is greater than the probability of the window breaking when the trajectory of the throw is not towards the window. 
Dispositions meet this condition. The probability that the window will break is greater if the glass is fragile. Furthermore, this condition has the advantage of capturing the intuitive idea that dispositions are tendencies which make certain outcomes more likely, rather than guaranteeing them.

Like the Counterfactual Dependence Condition, the Increased Probability Condition will still have some problems with co-extensive and closely related properties. The problem with closely related properties takes the following form. The ball's traveling $40 \mathrm{mph}$ is causally relevant to the window breaking. But suppose that the window would have been more likely to break if the ball had been traveling $50 \mathrm{mph}$. Now, $\mathscr{P}$ (breaking/traveling $40 \mathrm{mph}$ ) $<\mathscr{P}$ (breaking/traveling $50 \mathrm{mph}$ ). Since traveling $50 \mathrm{mph}$ is one way of not traveling $40 \mathrm{mph}$, traveling $40 \mathrm{mph}$ is not causally relevant, by the Increased Probability Condition. The condition is going to say that a property $\mathrm{F}$ is not causally relevant to e whenever there is a closely related property that would increase the probability of e to a greater extent than F does. What seems to be causing the trouble is that we need some way to determine what would follow if the cause differed by one property. What is c like when c does not have F? In some cases, you cannot just subtract F from c; c will have some other property instead.

Perhaps the details of this account could be worked out to overcome these problems. Despite these problems, the Increase Probability Condition could be part of a promising account that will allow causally relevant dispositions.

\section{The Independence Condition (Dardis 1993; Horgan 1989; Lepore and Loewer 1989; Ludwig 1998)}

Besides weighing one pound, and traveling $70 \mathrm{mph}$, Johnny's ball had another property. In retrospect, we can now say that it was a "windowbreaker." Something is a window-breaker at every point of its existence if, at any point of its existence, it breaks a window. Now, the mass and the velocity of the ball were relevant to the window breaking. What about being a window-breaker? Could it be causally relevant to the window breaking? The Independence Condition says "no." According to the Independence Condition, if $\mathrm{F}$ is causally relevant to e, there is no non-causal dependence between $\mathrm{F}$ and e. The F is not "committed" to causing e. In other words:

If $\mathrm{F}$ is causally relevant to $\mathrm{e}$, then there is a possible world in which c has F, but c does not cause e, $\&$ there is a possible world in which c causes e, but c does not have F. 
In every possible world in which Johnny's throw causes the window to break, Johnny throws a "window-breaker." So, being a window-breaker is not causally relevant to the window breaking.

Arguably, dispositions do not satisfy the Independence Condition. As mentioned, there is a conceptual dependence between the concept of a disposition and the concept of its manifestation. There is no possible world in which an attack is lethal, but does not kill. There is no possible world in which an attack kills, but is not lethal. So, being lethal is not independent of death, and so does not meet the Independence Condition. However, some dispositions might satisfy this condition if they are probilifying rather than sure-fire. Sometimes fragile things do not break when struck, and sometimes non-fragile things break when struck.

While some applications of the Independence Condition seem plausible, other consequences are counterintuitive. For example, sunburn is conceptually dependent on sunlight; there is no possible world in which an event causes sunburn, but that event is not an exposure to sunlight. Proponents of the Independence Condition claim that exposure to sunlight is not causally relevant to sunburn (Dardis 1993). More importantly, the Independence Condition rests on deep metaphysical assumptions about contingency of causal connections. On this view, any causal generalization "F-events cause Gevents" might have been false. This view is very contentious and is unlikely to be accepted without argument (Ellis 2001; Shoemaker 1980; Stalnaker 1987).

A further problem with this condition is that it seems to confuse semantics and metaphysics. We should distinguish relations between things in the world, and relations between our concepts. Finding a relation between our concepts does not preclude finding an interesting relation between the entities that fall under those concepts. As Davidson says, if c causes e we can dub c "the cause of e." We could then formulate the apparent conceptual truth "the cause of e causes e." However, this should not tempt us to think that the cause of e does not cause e (1980). Analogously, suppose that F is causally relevant to e. Now dub F "a property which is causally relevant to e." We can now say "a property which is causally relevant to e is causally relevant to e," but we should not therefore think that $\mathrm{F}$ is not causally relevant to e. 


\section{The Pattern Condition (Fodor 1989; MacDonald and MacDonald 1995; McLaughlin 2002; Segal and Sober 1991)}

Various conditions on causal relevance appeal to the natural laws. The most straightforward way in which laws figure in causal relevance is captured in the Pattern Condition, according to which a causally relevant property is part of a lawful pattern. The complete account looks like this:

If $\mathrm{F}$ is causally relevant to e, then

there is some $c$ that causes e,

$c$ is an $F$ \& e is a $G$

$\&$ it is a law that Fs cause Gs.

Not knowing enough physics, I do not know how to apply this condition to the example of Johnny breaking the window. Perhaps, in making a causal relevance claim, I am assuming that the property I mention figures in some causal law, even if I do not know what that law is. However, while I think being a fastball was relevant to the window breaking, I do not think that "being a fastball" is likely to figure in any causal law. The Pattern Condition suggests that, if $\mathrm{F}$ is a causally relevant property, the predicate " $\mathrm{F}$ " would be mentioned in an ideal articulation of the laws. However, the causal laws might turn out to be very specific, and mention only a few fundamental properties, not the things we talk about every day.

Do dispositions figure into causal laws? Probably, but it depends what the causal laws turn out to be. In any case, there is reason to be hesitant about accepting the Pattern Condition. Being a punch in the nose might not be mentioned in a causal law, but it seems causally relevant to the nosebleed. The Pattern Condition would imply that most of our causal relevance claims are false. If they are, so be it. However, it would be preferable to have an account of causal relevance that can make more sense of our causal explanations. We should look for a condition that is less restrictive.

\section{The Relaxed Pattern Condition (MacDonald And MacDonald 1995)}

A natural move is to somehow relax the pattern condition. We want the causally relevant property to be law-governed, but we do not want to demand that the laws explicitly mention that property. Perhaps we can capture this idea as follows. 
If $\mathrm{F}$ is causally relevant to $\mathrm{e}$, then

there is some c that causes e,

$\mathrm{c}$ is an $\mathrm{F}$ and an $\mathrm{H}$ \& $\mathrm{e}$ is a $\mathrm{G}$

$\&$ it is a law that Hs cause Gs.

Since the causal sequence (c causes e) is governed by a law (Hs cause Gs) and c since is an instance of both F and H, F is causally relevant to e. Some philosophers use this condition to try to show that mental properties are causally relevant because their instances are also instances of physical, nomological properties (Ehring 1999; MacDonald and MacDonald 1995).

Dispositions will count as causally relevant, on this view. Suppose there is some law such that certain molecular bonds break when subject to forces of a certain magnitude. That type of molecular bonding and fragility are both instantiated by the windowpane. So, the fragility is causally relevant to the breaking. More to the point, any time a disposition is instantiated by a cause, the disposition will count as causally relevant to the effect.

However, this account does not screen off any properties of the cause. As long as causation is law-governed, any property of the cause will count as causally relevant. Both the color of the baseball and the mass are jointly instanced, and the mass figures into causal laws. But this gives me no inclination to think the color is causally relevant. So, the Relaxed Pattern Condition is too relaxed. However, there might still be a way to use laws to formulate a plausible condition on causal relevance.

\section{The Sufficiency Condition}

When all of the causally relevant properties are instantiated, the laws ensure that the effect will occur. So, the set of causally relevant properties is a set of properties that is sufficient for the effect. This condition is also too permissive, but I introduce it by way of building up to a more plausible account. To put it more formally:

If $\mathrm{F}$ is causally relevant to $\mathrm{e}$, then

F is a member of a set of c's properties which is sufficient for e, given the laws.

F does not have to be actually mentioned in the laws. However F, and other properties in S, plus the laws, guarantee that e will occur. The pitcher throw- 
ing a fastball, together with the mass of the ball, the trajectory of its travel, etc., plus the laws, are sufficient for the breaking of the window, even though being a fastball is not likely to figure in the laws.

Dispositions will count as causally relevant on this view. The fragility of the window could be part of a set of properties that was sufficient for the window breaking. The obvious problem with this view is that you could get a sufficient set of properties by throwing in all of the properties of the cause. A set of properties that included the color of the ball, in addition to the mass, velocity, etc., could be sufficient for e. So, this account is also too permissive. However, it motivates the following two conditions.

\section{The Exclusion Condition (Kim 1989; Prior et al. 1982; Segal and Sober 1991)}

In deciding which properties are causally relevant, we want to exclude properties that are superfluous. A property would not be necessary for the effect if it were not part of a set of properties that was sufficient for the effect.

If $\mathrm{F}$ is causally relevant to e, then

there is no set of c's properties which is sufficient for e, given the laws, that does not include F.

$\mathrm{F}$ is a member of every sufficient set. If there is a sufficient set that does not include $\mathrm{F}$, then $\mathrm{F}$ is not causally relevant.

This condition might screen off dispositions. If dispositions are higher-order properties, then the causal basis of the disposition, plus the conditions, would make for a sufficient set, without the disposition. The Exclusion Condition rules out the possibility that the disposition and the causal basis are both causally relevant, and this is thought to be a good thing, because otherwise, the breaking would be over-determined by the window's properties. If we did not apply the Exclusion Condition, every time a disposition was manifest, the effect would be over-determined, and that seems odd (Block 1990). Proponents of Exclusion claim that there cannot be two independent complete causes for one event, except in cases of over-determination, and overdetermination is very rare.

However, the Exclusion Condition formulated above rules out any possibility of over-determining causally relevant properties, and in this respect it goes too far. Recall my noticing the bright flashing light. Since there is a sufficient set of properties that does not include the flashing, the flashing is irrelevant, and since there is a sufficient set of properties that does not include the 
brightness, the brightness is irrelevant. Proponents of the Exclusion Condition have to say over-determination by properties is impossible, or they have to allow for exceptions to the condition. The latter amounts to an admission that the Exclusion Condition is not necessary for causal relevance.

If the Exclusion Condition is not necessary for causal relevance, it is not at all clear when we should expect it to be satisfied. Over-determination by properties, or something very much like it, is much more common than proponents of Exclusion propose. Adapting an example from Stephen Yablo, suppose that a baseball going $70 \mathrm{mph}$ is causally relevant to the window breaking - it is a member of set of properties sufficient for breaking (1992). This set is sufficient even if it does not include going over $50 \mathrm{mph}$. Nevertheless, it seems that the baseball's going over $50 \mathrm{mph}$ could also be causally relevant to the glass breaking.

Certain properties of causes have close logical or nomological relations with one another: mathematical properties, determinables and determinates, supervenient properties and realizers, for example. The Exclusion Condition holds that members of one special privileged set of properties of the cause are relevant to the effect, and all other properties are irrelevant. We need a way to decide which properties of the cause are in the special set. Perhaps the most specific, or the most fundamental properties are the causally relevant ones. ${ }^{3}$ Like the Pattern Condition, this view would have the consequence that most of the causal relevance claims we make are false. So, the Exclusion Condition may screen off dispositions, but it will screen off most other properties too.

\section{The Minimal Sufficiency Condition (Fodor 1989)}

Fortunately, there is another way to restrict the Sufficiency Condition. To screen off superfluous properties, we insist that the sufficient set be as small as it can be, while still being sufficient. More formally,

If $\mathrm{F}$ is causally relevant to $\mathrm{e}$, then

$\mathrm{F}$ is a member of a set $S$ of $\mathrm{c}^{\prime} \mathrm{s}$ properties which is sufficient for e, given the laws \& no proper subset of $S$ is sufficient for e, given the laws.

Minimal Sufficiency will render the same verdict as Exclusion in many cases. Suppose properties $\mathrm{P}$ and $\mathrm{Q}$ are sufficient for e. What about property R? Exclusion will say that there is a sufficient set that does not include $R$, so $R$ is not causally relevant. Minimal Sufficiency will say, since $\{P, Q, R\}$ has a proper subset that is sufficient for e, inclusion in $\{\mathrm{P}, \mathrm{Q}, \mathrm{R}\}$ does not show causal relevance. 
However, there are important cases where Exclusion and Minimal Sufficiency will disagree. The conditions will disagree about the causal relevance of certain properties when there are distinct sets of properties that are each sufficient for an effect. Suppose $\{P, Q\}$ and $\{\mathrm{Q}, \mathrm{R}\}$ are each sufficient for e. Exclusion will have to say we have one of those rare cases of over-determination, or that either P or R must be irrelevant, since there is a sufficient set that does not include it. Minimal Sufficiency would say, unless either P, Q, or R is sufficient for e by itself, all are causally relevant, for each are members of minimally sufficient sets.

Minimal Sufficiency can account for over-determining causally relevant properties. The brightness of the light would be a member of a set of properties that was sufficient for attracting my attention, and the flashing would be a member of a different sufficient set. As long as they are members of minimally sufficient sets, they are both causally relevant. Furthermore, there is no privileged minimally sufficient set. The ball's going $70 \mathrm{mph}$ would be a member of a set of properties that is sufficient for the window breaking, and going over $50 \mathrm{mph}$ would be a member of a different minimally sufficient set. Both going $70 \mathrm{mph}$ and going over $50 \mathrm{mph}$ can be causally relevant. Minimal Sufficiency screens out irrelevant properties. A set of properties that includes the whiteness of the ball will always have a proper subset that is sufficient for the breaking. Yet Minimal Sufficiency can accommodate closely related properties that have equal claims on causal relevance.

The Minimal Sufficiency Condition will allow for causally relevant dispositions. There may be a set of properties that is sufficient for the window breaking that includes the molecular structure of the glass, and excludes fragility. However, there may be another sufficient set that includes fragility and excludes that particular molecular structure. Both the fragility and the molecular structure will count as causally relevant, on this view.

\section{Conclusion}

Let us reconsider the arguments with which we began. The Analyticity Argument assumes that a causally relevant property must satisfy the Independence Condition. The dependence between fragility and breaking was grounds for saying that fragility is not causally relevant to breaking. The Exclusion Argument assumes that causally relevant properties must satisfy the Strong Explanatory Condition, and the Exclusion Condition. Since there is a complete explanation and a sufficient set of properties that do not include fragility, fragility is deemed irrelevant. However, we have seen that these three conditions on causal relevance are objectionable for indepen- 
dent reasons. There are other accounts of causal relevance available. Some of them are quite attractive, and most of them allow that dispositions are causally relevant.

We considered several conditions on causal relevance: Weak and Strong Explanatory, Counterfactual Dependence, Increased Probability, Independence, Pattern, Relaxed Pattern, Sufficiency, Exclusion, and Minimal Sufficiency. Of these, the Independence, Pattern, Strong Explanatory and Exclusion conditions were the most likely to imply that dispositions are not causally relevant to their manifestations. However, we saw that these conditions on causal relevance are very stringent and rule out not only dispositions, but also other properties that intuitively seem causally relevant. All of the other conditions on causal relevance we have considered are compatible with the causal relevance of dispositions. Some of these conditions (Weak Explanatory, Relaxed Pattern, and Sufficiency) are too permissive; without added conditions, they would count apparently irrelevant properties as causally relevant. However, the Counterfactual Dependence, Increased Probability, and Minimal Sufficiency conditions offer promising, disposition-friendly approaches to analyzing causal relevance.

Admittedly, each condition has some outstanding problems and details need to be worked out to develop a full account of causal relevance. Disposition-friendly accounts are certainly in no worse a position than dispositionunfriendly accounts, and I think they are actually in a better position for the following reason. Disposition-friendly conditions face a few counterexamples where they seem to render the wrong verdict regarding the causal relevance of certain properties. One hopes that, with refinement, we will reach some reconciliation between our favorite account and our intuitions about certain cases. However, the problems with the disposition-unfriendly accounts will not disappear with refinement. The Exclusion Condition cannot account for over-determining properties, or different sets of properties with equal claims to causal relevance, for it is central to Exclusion that such things must be ruled out. Tinkering with the Independence Condition will not result in an account that will allow that sunlight is causally relevant to sunburn, or allow that any property is necessarily causally relevant to some effect, for Independence is designed to screen off such properties.

The situation with the Pattern Condition is somewhat different. With refinement it can overcome certain problems and counter-examples. However, I suggest that refining the Pattern Condition moves us in the direction of a disposition-friendly account, such as Minimal Sufficiency. 


\section{Notes}

1. John Gibbons offers a more familiar example: The whiteness of a pool ball can figure into explanations, particularly on a "per pay" pool table, where the white ball is the only one that automatically comes out after it is sunk (Gibbons 2001). Yet here again, the whiteness seems causally irrelevant.

2. Braun presents a counter-example to this variant analysis: "Betty listens to Wilma to determine whether Wilma is singing at the right pitch to shatter the glass. Betty has decided to sing if Wilma's pitch is not correct. So if Wilma's singing had not had that pitch, it would still cause the glass to shatter, by first causing Betty to sing" (Braun 1995). The pitch is causally relevant, but the counterfactual is false.

3. Kim (1991) claims something along these lines.

\section{References}

Allen, Colin: 1995, “It Isn't What You Think: A New Idea About Intentional Causation," Nous 29(1), 115-126.

Block, Ned: 1990, "Can the Mind Change the World?," in George Boolos (ed.), Meaning and Method: Essays in Honor of Hilary Putnam, Cambridge University Press, Cambridge, pp. 137-170.

Block, Ned: 1995, "Causation and Two Kinds of Laws," in Cynthia MacDonald (ed.), Philosophy of Psychology: Debates on Psychological Explanation, Blackwell, Cambridge, pp. 78-84.

Braun, David: 1995, "Causally Relevant Properties," Philosophical Perspectives: AI, Connectionism, and Philosophical Psychology 9, 447-475.

Dardis, Anthony: 1993, "Sunburn: Independence Conditions on Causal Relevance," Philosophy and Phenomenological Research LIII(3), 577-598.

Davidson, Donald: 1980, Essays on Actions and Events, Oxford University Press, Oxford.

Dretske, Frederick: 1988, Explaining Behavior, MIT Press, Cambridge, MA.

Ehring, Douglas: 1999, “Tropeless in Seattle: The Cure for Insomnia," Analysis 59(1), 19- 24.

Ellis, B. D.: 2001, Scientific Essentialism, Cambridge University Press, New York.

Fodor, Jerry: 1989, “Making Mind Matter More," Philosophical Topics 27, 59-79.

Gibbons, John: 2001, "Knowledge in Action," Philosophy and Phenomenological Research 63(3), 579-600.

Heil, John and A. Mele: 1991, "Mental Causes," American Philosophical Quarterly 28, 61-71.

Hitchcock, Christopher Read: 1993, “A Generalized Probabilistic Theory of Causal Relevance," Synthese $97,335-354$.

Horgan, Terrence: 1989, “Mental Quasation," Philosophical Perspectives: Philosophy of Mind and Action Theory 3, 47-76.

Jackson, Frank: 1995, "Essentialism, Mental Properties and Causation," Proceedings of the Aristotelian Society 95, 253-268.

Jackson, Frank and Phillip Pettit: 1990, "Program Explanation: A General Perspective," 
Analysis 50(2), 107-117.

Kim, Jaegwon: 1991, “Epiphenomenal and Supervenient Causation," in David M. Rosenthal (ed.), The Nature of Mind, Oxford University Press, New York, pp. 257-265.

Kim, Jaegwon: 1988, “Explanatory Realism, Causal Realism, and Explanatory Exclusion," Midwest Studies in Philosophy XII, 225-239.

Kim, Jaegwon: 1989, “Mechanism, Purpose, and Explanatory Exclusion,” Philosophical Perspectives: Philosophy of Mind and Action Theory 3, 77-108.

Kim, Jaegwon: 1990, “Explanatory Exclusion and the Problem of Mental Causation," in Enrique Villaneuva (ed.), Information, Semantics and Epistemology, Blackwell, Cambridge, pp. 36-56.

Lepore, Ernest and Barry Loewer: 1987, “Mind Matters,” Journal of Philosophy 84, 630- 642.

Lepore, Ernest and Barry Loewer: 1989, "More on Making Mind Matter," Philosophical Topics 17, 175-191.

Ludwig, Kirk: 1998, “Functionalism, Causation, and Causal Relevance," Psyche; online @ http://psyche.cs.monash.edu.au/v4/psyche-4-04-ludwig.html

MacDonald, Cynthia and Graham MacDonald: 1995, "How to be Psychologically Relevant," in Cynthia MacDonald (ed.), Philosophy of Psychology: Debates on Psychological Explanation, Blackwell, Cambridge, pp. 60-77.

McLaughlin, Brian P.: 2002, “Type Epiphenomenalism, Anomalism, and Causal Priority," Philosophical Perspectives.

Moliere: 1935, Le malade imaginaire, comédie-ballet . . avec une notice biographique, une notice littéraire et des notes explicatives par René Vanbourdolle, Librairie Hachette, Paris.

Prior, Elizabeth W., Robert Pargetter, and Frank Jackson: 1982, “Three Theses about Dispositions," American Philosophical Quarterly 19(3), 251-257.

Ruben, David-Hillel: 1994, “A Counterfactual Theory of Causal Explanation," Nous 28, 465-481.

Segal, Gabriel and Elliot Sober: 1991, “The Causal Efficacy of Content," Philosophical Studies 63, 1-30.

Shoemaker, Sydney: 1980, "Causality and Properties," in Peter van Inwagen (ed.), Time and Cause: Essays Presented to Richard Taylor, pp. 109-136.

Stalnaker, Robert: 1987, Inquiry, MIT Press, Cambridge, MA.

Yablo, Stephen: 1992, “Mental Causation,” The Philosophical Review 101(2), 245-280.

Department of Philosophy

University of Nebraska-Lincoln

1008 Oldfather Hall

P.O. Box 880321

Lincoln, NE 68588-0321 U.S.A.

Email jmckitrick2@unl.edu 